\title{
Allemagne, France, monde arabe : expériences et perspectives d'une jeune chercheuse en histoire croisée
}

\section{Esther Möller}

\section{(2) OpenEdition Journals}

Édition électronique

URL : https://journals.openedition.org/dhfles/3698

DOI : 10.4000/dhfles.3698

ISSN : 2221-4038

Éditeur

Société Internationale pour l'Histoire du Français Langue Étrangère ou Seconde

Édition imprimée

Date de publication : 1 janvier 2013

ISSN : 0992-7654

Référence électronique

Esther Möller, «Allemagne, France, monde arabe : expériences et perspectives d'une jeune

chercheuse en histoire croisée ", Documents pour l'histoire du français langue étrangère ou seconde [En ligne], 50 | 2013, mis en ligne le 01 janvier 2017, consulté le 31 mars 2023. URL : http:// journals.openedition.org/dhfles/3698; DOI : https://doi.org/10.4000/dhfles.3698

Ce document a été généré automatiquement le 31 mars 2023.

Tous droits réservés 


\title{
Allemagne, France, monde arabe : expériences et perspectives d'une jeune chercheuse en histoire croisée
}

\author{
Esther Möller
}

1 Quel est l'itinéraire et quelles sont les perspectives actuelles d'une jeune chercheuse allemande qui a choisi de travailler en histoire de l'enseignement des langues dans une région marquée par la francophonie et caractérisée tant par un pluriculturalisme que par un plurilinguisme ancien? Telle est la question qui m'était posée lors de la table ronde organisée dans le cadre des 25 ans de la SIHFLES au CIEP.

\section{D'une expérience du plurilinguisme vers l'écriture d'une histoire croisée libanaise}

2 Avant même d'entrer à la faculté j'avais fait, sans m'en rendre compte, mes premières découvertes scientifiques de la langue française: j'avais passé une année comme bénévole dans un centre d'accueil pour personnes handicapées près de Paris, où j'étais une parmi beaucoup d'autres étrangers. Ensemble nous faisions l'expérience des différents niveaux du français et du fait qu'un mot bien adapté dans un contexte (familier) ne l'est pas forcément dans un autre (officiel, professionnel) - un phénomène beaucoup moins courant en français qu'en allemand. Après cette expérience à la fois sociale et culturelle, j'ai poursuivi un programme d'études franco-allemand, appelé "Cursus intégré » et soutenu par "l'Université franco-allemande », une organisation qui soutient ce genre de formation binationale. Étudiant l'histoire, le français, la pédagogie et la philosophie, j'ai passé le DEUG et la licence à l'université de Dijon et mon examen final de maîtrise à l'université de Mainz (Mayence). Assez vite après mon examen j'ai décidé de ne pas devenir professeur de lycée, mais de me lancer dans la recherche et de faire une thèse en histoire - et de lier par cette expérience mon intérêt et passion pour la langue et la culture françaises et mon "nouvel amour ", le monde arabe. Lors d'un voyage au Liban avec une amie j'avais découvert les multiples 
références à la France dans ce pays arabe, jusqu'à la connaissance très développée du français par beaucoup de Libanais. J'ai donc décidé de consacrer ma thèse à la francophonie au Liban, et très vite j'ai constaté que le nombre et le poids des écoles françaises au Liban constituaient un aspect central et digne d'intérêt. Comme focus j'ai choisi la question de savoir comment l'idée de la mission civilisatrice française a été, à travers les écoles françaises, enseignée et adaptée, mais aussi reçue, approuvée et rejetée par les différents cercles libanais. Je me suis demandée comment des membres d'institutions aussi différentes que les congrégations religieuses présentes au Liban depuis le XVII siècle, la Mission laïque française créée en 1902, l'Alliance israélite universelle créée en 1860 et le Collège protestant français de Beyrouth créé en 1928 pouvaient attacher une telle importance à cette question, si leur conception de cette idée était différente - ou pas - et comment leur attitude s'était transformée par le contact avec le public libanais. Au Liban, le fait d'être allemande et non française m'a quelquefois aidée pour avoir accès aux archives des écoles et pour entrer en dialogue avec anciens professeurs et élèves sur "leurs expériences avec le français ». Ma recherche a pu montrer qu'il y avait beaucoup de similitudes, mais aussi des différences considérables entre l'idée de la mission civilisatrice des différentes écoles françaises, que cette idée a été reçue et adaptée à des fins multiples par les Libanais et que le public libanais des institutions éducatives françaises ne se limitait pas, et de loin, au milieu chrétien voire catholique.

3 Actuellement, je continue à m'intéresser beaucoup à la question de l'enseignement des langues, mais aussi à l'histoire des écoles françaises au Proche-Orient et j'essaye de m'orienter vers une perspective encore plus large en intégrant les écoles locales. Parallèlement, j'ai commencé à explorer un autre champ de recherche, à savoir l'histoire de l'aide humanitaire dans le monde arabe, en particulier les mouvements de la Croix Rouge et du Croissant Rouge. Curieusement ces deux champs de recherche sont plus liés que je ne le croyais au début: en effet, les mêmes personnes, européennes et arabes, étaient souvent celles qui s'engageaient dans le domaine de l'éducation aussi bien que dans celui de l'humanitaire. En complément de cela, je m'intéresse plus généralement à la recherche sur les contacts culturels et aux différentes théories développées pour les analyser : l'histoire croisée, terme mis à la mode par M. Werner et B. Zimmermann en 2003, correspond à une approche très utile pour analyser des transferts de systèmes et de matières d'enseignement ou d'aide humanitaire car elle cherche à dépasser les approches comparatistes en histoire culturelle et met l'accent sur les niveaux d'analyse en-dessous de l'État, comme la société civile ou les organisations internationales. Cet angle d'approche correspond aussi à l'intention de la théorie de la "gouvernementalité ", initialement développée par Michel Foucault, surtout utilisée en sciences politiques, mais qui devrait être davantage utilisée dans l'approche historique. Ainsi, des groupes d'acteurs comme des corps enseignants, des associations de professeurs ou des réseaux d'anciens élèves reçoivent un nouveau poids pour étudier l'impact politique et social de l'enseignement. En ce qui concerne ces questions dans le contexte extra-européen, l'approche d'Entangled History a été conçue par Shalini Randeria et Sebastian Conrad pour souligner les liens étroits entre " métropole » et « colonie » et leurs influences réciproques en différents domaines, et donc aussi dans celui de l'enseignement. Malheureusement cette approche reste, jusqu'à aujourd'hui, encore très vague et je souhaite contribuer à la développer davantage. En effet, cette théorie n'est pas encore aussi avancée que celle des Postcolonial Studies qui, à la suite de F. Fanon et d'E. Said, constitue un autre moteur de 
mes analyses : souligner l'impact des structures et dépendances coloniales au-delà des déterminations juridiques d'une colonie est particulièrement fructueux pour mes recherches sur le Liban qui n'a jamais été une colonie française, mais a néanmoins subi et, à un certain degré aussi, cherché une influence considérable de l'hexagone.

\section{Perspectives actuelles de recherche}

4 Dans les perspectives actuelles de recherche concernant l'enseignement des langues, et en particulier du français, je pense qu'un focus sur les deux pans de cet enseignement c'est-à-dire sur les enseignants ainsi que sur les enseignés (ce qui est particulièrement important pour les contextes coloniaux dominés par des relations d'asymétrie considérable) est bénéfique et nécessaire. Encore plus souvent que par le passé, nous devons nous efforcer, même si cela demande beaucoup d'efforts culturels, linguistiques et autres, de développer ce double regard. Dans le sens de «l'histoire croisée », cela implique non seulement celui qui enseigne et celui qui est enseigné, mais aussi le chercheur qui les étudie. En effet, notre rôle de chercheur doit toujours être pris en compte parce que, ayant le français comme langue maternelle, langue seconde ou troisième langue, notre relation à cette langue n'est pas sans influence sur notre recherche.

Ensuite, je pense que des études comparatives sont très importantes et devraient être développées davantage. En particulier dans le contexte de l'histoire coloniale qui m'intéresse, la comparaison d'initiatives éducatives occidentales et locales, la connaissance de leurs similitudes et différences, mais aussi leurs processus d'adaptation et de rejet réciproques est primordiale pour comprendre les unes et les autres et pour cerner leur rôle central pour des États et sociétés en transformation politique, sociale et culturelle. Cela inclut aussi une approche plurielle des archives à consulter. Comme j'essaie de le faire dans mon nouveau projet de recherche, une exploitation et analyse comparative d'archives européennes et non européennes, religieuses et laïques, publiques et privées ne serait-elle pas un des moyens possibles pour mettre en pratique certains des concepts mis en avant ces dernières années par les théoriciens présentés plus haut? Par exemple une combinaison des archives françaises et indigènes pourrait aider à faire avancer - ou falsifier - les intentions de l'Entangled History, car peut-être, dans le domaine de l'enseignement des langues par exemple, les impacts des colonies sur la métropole ne sont pas toujours aussi flagrants que l'on aimerait les avoir.Grâce aux nouvelles possibilités offertes par les techniques de l'information et de la communication, davantage de matériel archivistique peut être recueilli et par la suite travaillé de manière comparative. Les nouvelles techniques favorisent un travail en équipe malgré l'éloignement des chercheurs et invitent donc à des projets de coopération dont les résultats peuvent également être présentés sous forme de plateformes interactives, de blogs thématiques ou de publications en ligne qui intègrent des photographies, des documents audiovisuels ou d'une autre nature encore sous-représentée dans les études actuelles. 


\section{AUTEUR}

\section{ESTHER MÖLLER}

Institut d'histoire européenne, Mayence 